\title{
Comparison of four different Shear Wave Elastography platforms according to abdominal wall thickness in liver fibrosis evaluation: a phantom study
}

\author{
Sang Min Lee, ${ }^{1,2}$, Won Chang ${ }^{3}$, Hyo-Jin Kang', Su Joa Ahn', Jeong-Hoon Lee ${ }^{4}$, Jeong Min \\ Lee $^{1,5,6}$
}

${ }^{1}$ Department of Radiology, Seoul National University Hospital, ${ }^{2}$ Department of Radiology, Hallym University Sacred Heart Hospital, ${ }^{3}$ Departement of Radiology, Seoul National University Bundang Hospital, ${ }^{4}$ Department of Internal Medicine, Seoul National University Hospital, ${ }^{5}$ Department of Radiology, Seoul National University College of Medicine, ${ }^{6}$ Institute of Radiation Medicine, Seoul National University Medical Research Center, Republic of Korea

\begin{abstract}
Aims: To compare the applicability, reliability and stiffness values of four different shear wave elastography (SWE) platforms and to determine the influence of abdominal wall thickness on those of four SWE platforms evaluating liver fibrosis. Material and methods: We used four different SWE platforms: transient elastography (TE), pSWE (S-shear wave), 2D SWE/ SSI (SuperSonic Imagine) and 2D SWE/GE (GE Health care). To identify the effect of abdominal wall thickness, five commercially available liver fibrosis phantoms were covered in $1.3 \mathrm{~cm}$ and $2.3 \mathrm{~cm}$ thick porcine abdominal walls, respectively. All measurements were performed by three observers. Absolute repeatability of each measurement was evaluated using coefficients of variation (CVs). Applicability rate, CVs and mean stiffness values were compared according to platforms and abdominal wall thickness. Results: Applicability rates were significantly different among the four SWE platforms ( $<<0.001): 2 \mathrm{D}$ SWE/SSI showed the lowest applicability rate (68.9\%) compared with the other three platforms (TE, 95.6\%, pSWE, 93.3\%, 2D SWE/GE, 97.8\%) due to higher technical failure rates in phantoms with thick wall. Repeatability was significantly different according to the platform and abdominal wall thickness. Stiffness values did not significantly differ according to abdominal wall thickness, even though the values were significantly different among four platforms in all phantoms. Conclusions: The applicability, repeatability, and stiffness value were different depending on SWE platforms. Further, the applicability and repeatability would be more affected by abdominal wall thickness. Thus, careful consideration should be given to an appropriate SWE platform, when evaluating liver fibrosis in patients with thick abdominal wall.
\end{abstract}

Keywords: shear wave elastography; liver stiffness measurements; abdominal wall thickness

\section{Introduction}

Liver fibrosis is the most important predictor of disease outcome in chronic viral hepatitis, and thus, accurate

Received 02.10.2018 Accepted 11.11.2018

Med Ultrason

2019, Vol. 21, No 1, 22-29

Corresponding author: Jeong Min Lee, M.D.

Department of Radiology, Seoul National

University Hospital, 101 Daehangno,

Jongno-gu, Seoul, 110-744, Republic of Korea

E-mail: jmsh@snu.ac.kr assessment of the extent of hepatic fibrosis is crucial for the selection of the most appropriate therapeutic strategy and for monitoring the responses to anti-viral treatment [1-3]. Indeed, the need for accurate fibrosis staging is of great clinical importance, as new direct-acting antiviral agents are only indicated in patients with advanced fibrosis or cirrhosis in many countries [3-5]. Until now, liver biopsy has been considered to be the reference standard for the evaluation of liver fibrosis; however, it has been documented to have several clear limitations including its invasiveness, sampling variability, and/or intra- and inter-observer variability [6-8]. With this background, 
advancements in non-invasive methods such as transient elastography (TE) has rapidly replaced percutaneous liver biopsy for both the assessment of liver disease severity and the management of patients with chronic liver diseases (CLD) [8,9].

Among the non-invasive techniques, TE which is a one-dimensional ultrasound system that induces an external mechanical vibration which propagates through the liver, with faster movement indicating a stiffer liver [10], has been the most widely validated non-invasive tool for the detection of liver fibrosis, with good reproducibility and good diagnostic value in liver fibrosis [11-14]. More recently, other elastographic techniques including magnetic resonance (MR) elastography and ultrasound (US) based point shear wave elastography (pSWE) and two-dimensional shear wave elastography (2D SWE) have also been developed [9,15-17]. In the pSWE technique [Virtual Touch Quantification (VTQ; Siemens, Berlin, Germany) ElastPQ (Philips Medical Systems, Bothell, WA, USA), S-shearwave (Samsung Medison, Seoul, Korea)], a single focused acoustic radiation force pushing pulse with a short duration ( 262 $\mu \mathrm{sec})$ generates one shear wave with localized displacement in tissue using the same probe as that of a conventional diagnostic US system [18]. The more recently developed 2DSWE techniques [real-time SWE (Supersonic Imagine [SSI], Aix en Provence, France), 2D SWE (GE Healthcare [GE], Milwaukee, WI, USA), ElastQ (Philips Medical Systems) and continuous SWE (Canon Medical Systems, Otawara-shi, JAPAN)], on the other hand, enables instant qualitative evaluation and accurate quantitative evaluation of tissue elasticity by providing real-time colored stiffness maps and a large field-of-view (FOV) on commercial US systems [10,14].

Until now, there have only been a few studies comparing two or more elastography platforms [19]. As applicability and reliability can vary depending on the study population as well as which elastography platforms are used, it would be meaningful to evaluate the applicability and reliability of various elastography platforms in the same population. Moreover, there have been few studies examining the influence of abdominal wall thickness to the applicability, reliability and measurement of stiffness values with the differing SWE platforms. Therefore, the purpose of our study was to compare the applicability, reliability, and stiffness values among four SWE platforms including TE, pSWE and two types of 2D SWE (SSI and GE) using phantoms, and to assess whether abdominal wall thickness influences the applicability, reliability and stiffness values at each SWE platform using 15 phantoms covered with different level of porcine abdominal wall thickness.

\section{Material and methods}

\section{Phantoms}

To evaluate the applicability, reliability, and stiffness values of the four SWE platforms, 15 phantoms were used. As a control group, we used five commercially available liver fibrosis phantoms (\#1 \#5), filled with a polymer gel mimicking tissue (Zerdine) formulated with differing values of Young's modulus (Computerized Imaging Reference System Company [CIRS], Norfolk, VA, USA). The actual values of Young's modulus of each phantom were not provided by the manufacturer. The mean stiffness values (mean \pm standard deviation) on $\mathrm{TE}$ for the original phantoms (\#1 \#5) measured by the three observers were $3.6 \pm 0.0 \mathrm{kPa}, 14.5 \pm 0.3 \mathrm{kPa}, 16.1 \pm 0.2 \mathrm{kPa}$, $16.7 \pm 0.1 \mathrm{kPa}$ and $18.4 \pm 0.4 \mathrm{kPa}$, respectively. The phantom was $10 \mathrm{~cm}$ wide and $12 \mathrm{~cm}$ deep and was contained in a $14 \mathrm{~cm}$ high cylinder. The density of the phantom was $1.03 \mathrm{~g} / \mathrm{cm} 3$. In addition, in order to evaluate the influence of abdominal wall thickness in stiffness measurements on SWE platforms, we created additional phantoms by placing porcine abdominal walls of different thicknesses consisting of dermis, subcutaneous fat layer and muscle layer similar to human abdominal wall on the commercial phantoms. In total, 15 different phantom subjects, consisting of 5 original phantoms, 5 phantoms with thin abdominal wall (original phantoms covered with $1.3 \mathrm{~cm}$ thick porcine tissue, \#6 \#10) and 5 phantoms with thick abdominal wall (original phantoms covered with $2.3 \mathrm{~cm}$ thick porcine tissue, \#11 \#15) were included (fig 1).

\section{Stiffness measurements}

We measured stiffness values of the 15 different phantoms ( 5 original phantoms, 5 phantoms with thin wall, and 5 phantoms with thick wall) using four SWE platforms including TE (FibroScan, Echosens, Paris, France), pSWE (RS80A with Prestige, Samsung Medison, Seoul, Korea), 2DSWE/SSI (Aixplorer, SuperSonic Imagine, Aix-en-Provence, France) and 2D SWE/GE (Logiq E9, GE healthcare, Milwaukee, WI, USA). Three radiologists (S.M.L., W.C., and H.J.K., with 9, 7 and 5 years of experience in abdominal ultrasound, respectively) measured the 15 different phantoms using pSWE, 2D SWE/SSI and 2DSWE/GE platforms (fig 2). One radiologist (S.M.L.) and two technicians (Y.H.L. and M.R.L., with 3 years of experience in TE) performed TE for all 15 phantoms. The observers were blinded to the results obtained by the other observers. For intra-observer variability, one radiologist (S.M.L.) measured the 15 phantoms on TE and the three SWE platforms twice, with a one-day interval. A teach session, 10 sequential stiffness values were measured, from which the median stiffness value, the mean stiffness value, and its standard deviation (SD) 


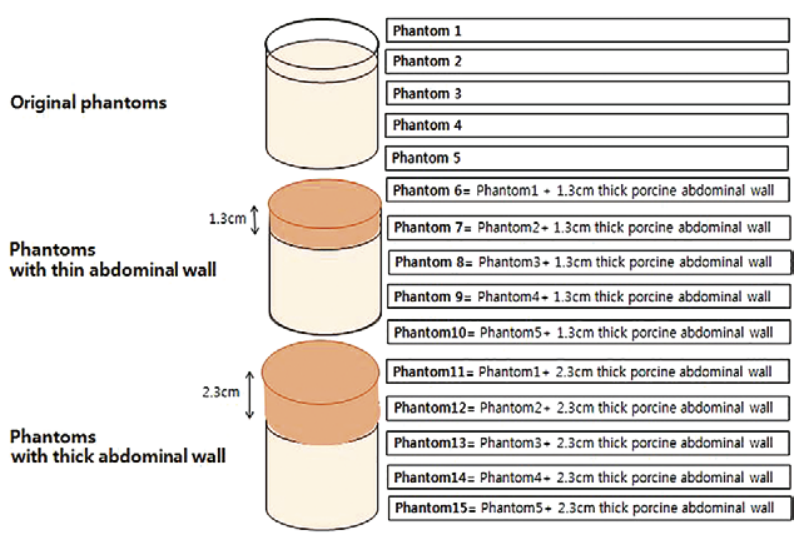

Fig 1. Phantoms.A total of 15 different phantom subjects consists of 5 original phantoms, 5 phantoms with a thin abdominal wall (original phantoms covered with $1.3 \mathrm{~cm}$ thick porcine tissue, \#6 \#10)), and 5 phantoms with a thick abdominal wall (original phantoms covered with $2.3 \mathrm{~cm}$ thick porcine tissue, \#11 \#15).

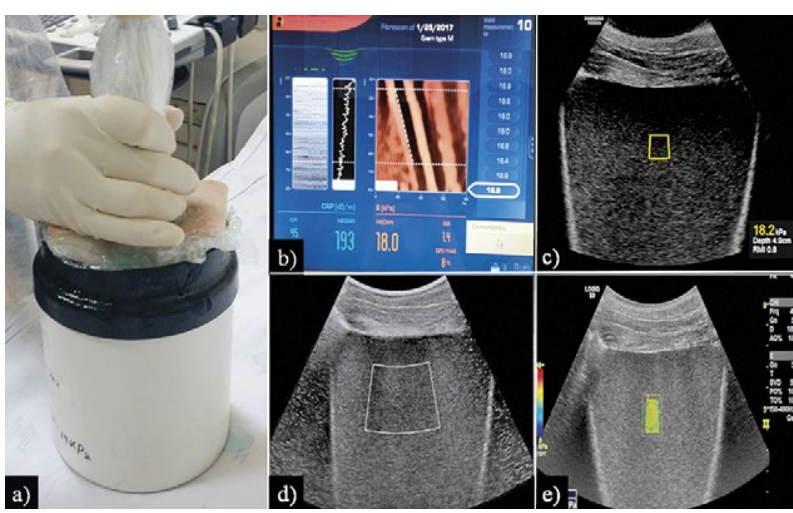

Fig 2. Measurement of stiffness values in phantoms using four elastography platforms: a) Placement of a convex probe on stiffness phantom \#14 (phantom \#4 covered in the $2.3 \mathrm{~cm}$ thick porcine abdominal wall) using 2D SWE/GE (LOGIQ E9, GE Healthcare; b-e) Measurements of stiffness values for phantom \#13 using four different elastography platforms including TE(FibroScan, Echosens), pSWE (RS80A with Prestige, Samsung Medison), 2D SWE/SSI (Aixplorer, SuperSonic Imagine), and 2D SWE/GE. The elastographic graph and stiffness values obtained by TE with an M probe (b) are shown. Regions of interest (ROIs) were placed $1.5-2 \mathrm{~cm}$ away from the phantom surface on pSWE (c), 2D SWE/SSI (d), and 2D SWE/GE (e). Technical failure was observed on 2D SWE/SSI.

were obtained. Stiffness values were expressed in $\mathrm{kPa}$ in all platforms. Median stiffness values were taken as the representative result for comparison of stiffness values. To evaluate the repeatability of measurements which could be regarded as an absolute reliability indicator, coefficients of variation $(\mathrm{CVs})$ for each measurement were also calculated using the following equation: $\mathrm{CV}=\mathrm{SD} /$ mean stiffness value [20]. A lower CV indicates higher repeatability of each measurement $[20,21]$.

\section{Transient Elastography (TE)}

We performed TE using a 3.5-MHx "M" probe, which is the standard probe recommended for a measurement depth between 25-65 $\mathrm{mm}$ [22]. The tip of the probe was placed at the center of the phantom surface with minimum compression using the free-hand technique. The location could be adjusted using a time-motion image. Thereafter, the observer pressed the "shot" button of the transducer attached to a mechanical vibrator to provide an external punch pulse. The median value from 10 repeated valid measurements was expressed in $\mathrm{kPa}$ on the screen. The software then determined whether it was a valid measurement for each measurement and displayed it on the screen [17].

\section{Point Shear Wave Elastography (pSWE)}

When using the pSWE technique implemented on RS80A with Prestige, also referred to as S-shear wave, the convex probe (CA1-7A), similar to that for an abdominal ultrasound scan, was held with minimum pressure on the center of the phantom surface. Under B-mode image guidance, a fixed region-of-interest (ROI) was placed $1.5-2 \mathrm{~cm}$ away from the phantom surface in the same location for the 10 repeated valid measurements. The pSWE platform did not report stiffness values when measurements were invalid.

\section{D Shear Wave Elastography:}

SuperSonic Imagine (SSI) and GE healthcare

We used two types of 2D SWE with different mechanisms to generate the shear waves and to track the shear waves in real-time. With 2DSWE/SSI, a shear wave was generated using push beams in a multiple focal zone configuration, and a software beamformer enabled tracking of the shear waves at a fast frame rate [23]. The 2DSWE/ $\mathrm{GE}$, on the other hand, used the comb-push technique to produce shear waves with a comb pattern and timealigned sequential tracking to catch the shear wave signal at a faster frame rate with parallel beamforming capability on a conventional US system [24-26].

The observers held the convex probe (SC6-1 probe for SSI and C1-6 probe for GE) with minimum compression on the surface of the phantom. The colored FOV box $\left(\sim 2 \mathrm{~cm}^{2}\right)$ was set up for placement $1.5-2 \mathrm{~cm}$ away from the phantom surface at the same location for the 10 repeated measurements. A $1 \times 1 \mathrm{~cm}^{2}$ circular region-of-interest (ROI, named Q-Box ${ }^{\mathrm{TM}}$ in SSI), which was adjustable in size and position, was placed in areas of the measured FOV box that displayed homogeneous color filling. No or inadequate color mapping indicated an invalid measurement.

Technical failure was defined as when a valid measurement or a proper color map with less than $30 \%$ artifacts in the sampling area was not obtained in 10 trials 
$[17,27]$. When the interquartile range (IQR)/median ratio of the 10 measurements was higher than $30 \%$, the data was regarded as unreliable [28]. The applicability rate was defined as the ratio of exams with technical success and reliable measurements and all exams [29].

\section{Statistical analysis}

Statistical analyses were performed using commercially available software programs (SPSS, version 23, IBM, Armonk, NY; or MedCal, version 16, MedCalc software, Mariakerke, Belgium). We used the $\chi^{2}$ or Fisher's exact test to determine whether the applicability rate differed depending on SWE platforms and different level of abdominal wall thickness. Intra-observer and inter-observer reproducibility were determined using intraclass correlation (ICC). For each case consisting of 10 measurements, a coefficient of variation $(\mathrm{CV}$; standard deviation / average) showing absolute measurement repeatability was obtained and tested for normality using the Kolmogorov-Smirnov test. The Kruskal-Wallis test was performed to compare the CVs among the four different SWE platforms and three different level of abdominal wall thickness. To compare stiffness values among all platforms and different level of abdominal wall thickness, the Kruskal-Wallis test was used. A p value of less than 0.05 was considered to indicate a significant difference. On post hoc analysis for multiple comparisons, the $\mathrm{p}$ value was corrected using Bonferroni's method.

\section{Results}

\section{Applicability rate}

The applicability rate for all phantoms was significantly greatest in the 2D SWE/GE $(97.8 \%, 44 / 45)$, followed by TE $(95.6 \%, 43 / 45)$, pSWE $(93.3 \%, 42 / 45)$ and 2D SWE/SSI $(68.9 \%, 31 / 45 ; \mathrm{p}<0.001)$ (Table I). On post-hoc analysis using Bonferroni adjusted $\mathrm{p}$ values $(\mathrm{p}=0.005 / 6=0.008)$, the applicability rate of 2D SWE/SSI was significantly lower than TE, pSWE and 2D SWE/GE ( $\mathrm{p}=0.001, \mathrm{p}=0.003, \mathrm{p}<0.001$, respectively).

There were no cases of technical failure nor unreliable data in any of the four SWE platforms, showing $100 \%$ applicability for original phantoms (\#1-\#5) without porcine abdominal wall. However, the applicability of all SWE platforms for phantoms (\#6-\#10) with a thin $(1.3 \mathrm{~cm})$ porcine abdominal wall and phantoms (\#11\#15) with a thick $(2.3 \mathrm{~cm})$ porcine abdominal wall were $96.7 \%$ and $70.0 \%$, respectively. There were significantly different applicability rates among all SWE platforms for phantoms with a thick porcine abdominal wall $(\mathrm{p}<0.001)$. The applicability rates of pSWE $(p=0.004)$ and 2D SWE/ SSI $(p<0.001)$ were significantly different according to the level of abdominal wall thickness, while those of TE $(\mathrm{p}=0.123)$ and $2 \mathrm{D} \mathrm{SWE} / \mathrm{GE}(\mathrm{p}=0.36)$ did not show a significant difference.

In addition, the applicability rate of the four SWE platforms in the three observers was $88.3 \%, 88.3 \%$, and $90.0 \%$, respectively, with no significant difference $(\mathrm{p}=0.945)$.

\section{Reproducibility and repeatability}

All platforms provided high ICCs for intra-observer reproducibility, ranging from 0.97-0.99 (Table II). The ICC for inter-observer reproducibility was 0.99 in all platforms. Although the pooled CV was less than 1.0 in all platforms, the CVs were significantly different in pSWE (0.068), 2D SWE/ GE (0.067), TE (0.051) and 2D SWE/SSI $(0.030)(p<0.001$, Table III). For original phantoms, 2D SWE/SSI showed lowest CV (0.012), indicating best repeatability among four SWE platforms. CVs were significantly different in phantoms with thick porcine abdominal wall (all $\mathrm{p}<0.05$ ). CVs in all platforms, except for 2D SWE/SSI, tended to increase as wall thickness increased.

\section{Stiffness value}

Mean stiffness values for all 15 phantoms measured in TE, pSWE, 2D SWE/SSI and 2D SWE/GE were $15.1 \mathrm{kPa}, 16.2 \mathrm{kPa}, 15.6 \mathrm{kPa}$ and $13.3 \mathrm{kPa}$, respectively ( $\mathrm{p}=0.001$, Table IV). Post hoc analysis using the Mann-Whitney $U$ test revealed that the mean stiffness value in $2 \mathrm{D} \mathrm{SWE} / \mathrm{GE}$ was significantly lower than those of pSWE and 2D SWE/SSI ( $<0.001, \mathrm{p}=0.002)$, while the value in $2 \mathrm{D} \mathrm{SWE} / \mathrm{GE}$ was not significantly different from that of TE $(\mathrm{p}=0.027$, using a significance level of $\mathrm{p}=0.008$, Bonferroni's method).

Mean stiffness values of original phantoms measured by the four SWE platforms were not different from those of phantoms with thin porcine wall and thick wall (all $p>0.05$ ). In addition, the mean stiffness values of the 15 phantoms in all of the four SWE platforms measured by the three observers were not significantly different $(\mathrm{p}=0.915)$.

\section{Discussions}

US-based SWE platforms have been widely used as a tool to measure liver stiffness values, and thus nearly all US manufacturers have developed their own SWE platforms [9]. Although these US-based SWE platforms use the same acoustic radiation force impulse imaging (ARFI) technique to generate shear waves, different mechanisms to detect the shear waves in real-time are implemented on each commercial US system [16]. Currently, it remains unclear whether the measured stiffness values using these US-based SWE platforms are interchangeable, and yet few studies have compared the re- 
Table I. Applicability rate (\%) of each elastography platform

\begin{tabular}{|c|c|c|c|c|c|c|}
\hline & TE & pSWE & 2D SWE/SSI & 2D SWE/GE & All platforms & p valuet \\
\hline Original phantoms & $100(15 / 15)$ & $100(15 / 15)$ & $100(15 / 15)$ & $100(15 / 15)$ & $100(60 / 60)$ & 1 \\
\hline Phantoms with thin abdominal wall & $100(15 / 15)$ & $100(15 / 15)$ & $86.7(13 / 15)$ & $100(15 / 15)$ & $96.7(58 / 60)$ & 0.102 \\
\hline Phantoms with thick abdominal wall & $86.7(13 / 15)$ & $80(12 / 15)$ & $20(3 / 15)$ & $93.3(14 / 15)$ & $70(42 / 60)$ & $<0.001$ \\
\hline All phantoms & $95.6(43 / 45)$ & $93.3(42 / 45)$ & $68.9(31 / 45)$ & 97. $(44 / 45)$ & $88.9 \%(160 / 180)$ & $<0.001$ \\
\hline $\mathrm{p}$ value & 0.123 & 0.004 & $<0.001$ & 0.36 & $<0.001$ & \\
\hline
\end{tabular}

The results are expressed as percent (exams with technical success and reliable measurements/ all exams) ; †Comparison of applicability rates between four elastography platforms; $\$$ Comparison of applicability rates between different levels of abdominal wall thickness at each elastography platform.; TE, transient elastography; pSWE, point shear wave elastography; 2D SWE/SSI, 2D-shear wave elastography - SuperSonic Imagine; 2D SWE/GE, 2D-shear wave elastography - GE Healthcare

Table II. Reproducibility at each elastography platform

\begin{tabular}{lllll}
\hline & \multicolumn{2}{l}{ ICC for intraobserver variability } & \multicolumn{2}{l}{ ICC for interobserver variability } \\
\cline { 2 - 5 } & ICC & $\mathbf{9 5 \%}$ CI & ICC & $0.979,0.997$ \\
TE & 0.996 & $0.987,0.999$ & 0.992 & $0.991,0.999$ \\
$\mathrm{pSWE}$ & 0.979 & $0.934,0.993$ & 0.996 & $0.991,0.999$ \\
2D SWE/SSI & 0.997 & $0.989,0.999$ & 0.997 & $0.966,0.995$ \\
2D SWE/GE & 0.971 & $0.911,0.991$ & 0.986 & \\
\hline
\end{tabular}

ICC, intraclass correlation; TE, transient elastography; pSWE, point shear wave elastography; 2D SWE/SSI, 2D-shear wave elastography SuperSonic Imagine; 2D SWE/GE, 2D-shear wave elastography - GE Healthcare

Table III. Coefficient of variation (CV) at each elastography platform

\begin{tabular}{|c|c|c|c|c|c|c|}
\hline & TE & pSWE & 2D SWE/SSI & 2D SWE/GE & All platforms & p valuet \\
\hline $\begin{array}{l}\text { Original } \\
\text { phantoms }\end{array}$ & $\begin{array}{l}0.028 \\
(0.00-0.062)\end{array}$ & $\begin{array}{l}0.052 \\
(0.025-0.092)\end{array}$ & $\begin{array}{l}0.012 \\
(0.005-0.025)\end{array}$ & $\begin{array}{l}0.025 \\
(0.00-0.038)\end{array}$ & $\begin{array}{l}0.029 \\
(0.00-0.092)\end{array}$ & $<0.0001$ \\
\hline $\begin{array}{l}\text { Phantoms } \\
\text { with thin abdominal wall }\end{array}$ & $\begin{array}{l}0.059 \\
(0.014-0.134)\end{array}$ & $\begin{array}{l}0.065 \\
(0.030-0.106)\end{array}$ & $\begin{array}{l}0.050 \\
(0.012-0.213)\end{array}$ & $\begin{array}{l}0.056 \\
(0.00-0.119)\end{array}$ & $\begin{array}{l}0.058 \\
(0.00-0.213)\end{array}$ & 0.107 \\
\hline $\begin{array}{l}\text { Phantoms } \\
\text { with thick abdominal wall }\end{array}$ & $\begin{array}{l}0.069 \\
(0.015-0.193)\end{array}$ & $\begin{array}{l}0.097 \\
(0.029-0.159)\end{array}$ & $\begin{array}{l}0.036 \\
(0.033-0.041)\end{array}$ & $\begin{array}{l}0.119 \\
(0.018-0.522)\end{array}$ & $\begin{array}{l}0.092 \\
(0.015-0.522)\end{array}$ & 0.156 \\
\hline $\begin{array}{l}\text { All } \\
\text { phantoms }\end{array}$ & $\begin{array}{l}0.051 \\
(0.00-0.193)\end{array}$ & $\begin{array}{l}0.068 \\
(0.025-0.159)\end{array}$ & $\begin{array}{l}0.030 \\
(0.005-0.213)\end{array}$ & $\begin{array}{l}0.067 \mid \\
(0.00-0.522)\end{array}$ & $\begin{array}{l}0.056 \\
(0.00-0.522)\end{array}$ & $<0.0001$ \\
\hline $\mathrm{p}$ value & 0.018 & 0.013 & $<0.0001$ & 0.001 & $<0.0001$ & \\
\hline
\end{tabular}

The results are expressed as mean CVs (range). $\uparrow$ Comparison of CVs for all phantoms between four elastography platforms using the Kruskal-Wallis test; †Comparison of CVs between different levels of abdominal wall thickness at each elastography platform; TE, transient elastography; pSWE, point shear wave elastography; 2D SWE/SSI, 2D-shear wave elastography - SuperSonic Imagine; 2D SWE/GE, 2Dshear wave elastography - GE Healthcare

Table IV. Stiffness values of phantoms at each elastography platform

\begin{tabular}{|c|c|c|c|c|c|c|}
\hline & TE & pSWE & 2D SWE/SSI & 2D SWE/GE & All platforms & p valuet \\
\hline $\begin{array}{l}\text { Original } \\
\text { phantoms }\end{array}$ & $13.9 \pm 5.6$ & $16.4 \pm 6.3$ & $15.2 \pm 5.8$ & $14.1 \pm 5.3$ & $14.9 \pm 5.7$ & 0.056 \\
\hline $\begin{array}{l}\text { Phantoms } \\
\text { with thin abdominal wall }\end{array}$ & $15.9 \pm 7.1$ & $16.1 \pm 6.4$ & $15.4 \pm 6.3$ & $12.3 \pm 4.3$ & $14.9 \pm 6.1$ & 0.029 \\
\hline $\begin{array}{l}\text { Phantoms } \\
\text { with thick abdominal wall }\end{array}$ & $15.7 \pm 7.4$ & $15.9 \pm 6.6$ & $18.9 \pm 1.5$ & $13.5 \pm 5.1$ & $15.2 \pm 6.7$ & 0.058 \\
\hline $\begin{array}{l}\text { All } \\
\text { phantoms }\end{array}$ & $15.1 \pm 6.6$ & $16.2 \pm 6.2$ & $15.6 \pm 5.8$ & $13.3 \pm 4.9$ & $15.0 \pm 5.9$ & 0.001 \\
\hline $\mathrm{P}$ value & 0.177 & 0.877 & 0.294 & 0.243 & 0.622 & \\
\hline
\end{tabular}

The results are expressed asmean \pm standard deviation $(\mathrm{kPa})$. ; †Comparison of mean stiffness values for all phantoms between four elastography platforms using the Kruskal-Wallis test; $\$$ Comparison of mean stiffness values between different levels of abdominal wall thickness at each elastography platform; TE, transient elastography; pSWE, point shear wave elastography; 2D SWE/SSI, 2D-shear wave elastography - SuperSonic Imagine; 2D SWE/GE, 2D-shear wave elastography - GE Healthcare 
producibility of these differing SWE platforms [30-32]. In addition, there had not yet been any evaluation regarding differences in their applicability, reproducibility, and stiffness values of different SWE platforms according to abdominal wall thickness. For this purpose, we performed a comparison between TE, pSWE, 2D SWE/SSI and 2D SWE/GE in 15 different tissue-mimicking stiffness phantoms covered with three levels of porcine abdominal wall thickness and demonstrated that the applicability rates were significantly different among the four SWE platforms and applicability. Specifically, among the four platforms, 2D SWE/SSI showed a lower applicability rate $(68.9 \%)$ compared with the other three platforms due to higher technical failure rates in phantoms with thick abdominal wall. In addition, although all four SWE platforms showed excellent intra-and inter-observer reproducibility, absolute repeatability expressed in $\mathrm{CV}$ was significantly different according to platforms and porcine abdominal wall thicknesses. However, the mean stiffness values did not significantly differ according to abdominal wall thickness in each SWE platform, even though the values obtained by the four SWE platforms were significantly different.

In our study, we found that 2D SWE/SSI may be highly influenced in terms of applicability according to abdominal wall thickness, which is consistent with previous research comparing 2D SWE/SSI, pSWE and TE performed by Cassinotto et al [19]. They demonstrated that 2D SWE/SSI the technical failure of 2D SWE/SSI was associated with obesity and intercostal wall thickness. This can be explained by the fact that there is a reduction of shear wave propagation when the soft tissue is thick and 2D SWE/SSI seems to be particularly affected by this phenomenon [19]. Therefore, according to our study results, in patients with thick abdominal wall, TE, pSWE or 2D SWE/GE may be more suitable for the measurement of stiffness value than 2D SWE/SSI.

Excellent intra- and inter-observer reproducibility were demonstrated in all four SWE platforms in our study. Our study results are similar to that of a previous study using three SWE platforms and strain elastography [33]. However, we found pSWE showed highest CV, suggesting the lowest absolute repeatability. Despite having the lowest applicability, 2D SWE/SSI showed the lowest $\mathrm{CV}$ (the greatest absolute repeatability) among the four SWEs. This result is concordant with that of a previous study comparing 2D SWE/SSI and two kinds of pSWE using a homogenous stiffness phantom of $16.9 \mathrm{kPa}$ [21], in which the CVs of 2D SWE/SSI (0-0.03) were low, as in our results. Furthermore, we demonstrated that the CVs in all platforms, except for 2D SWE/SSI, tended to increase as the abdominal wall thickness increased, sug- gesting that abdominal wall thickness may affect applicability and repeatability. Although there have been several reports regarding the influence of measurement depth [21,34-35], until now there has been no report regarding the influence of abdominal wall thickness.

Our study showed a significant difference in mean stiffness values for all phantoms obtained using the four SWEs. On post hoc analysis, the three SWE stiffness values were not shown to be different from TE values, but 2D SWE of GE showed lower values than pSWE and 2D SWE/SSI. This result is discordant with a previous study in which the stiffness values of 2D SWE/SSI and 2D SWE/GE were shown to be similar [33]. In addition, this result is inconsistent with that of a phantom experiment by Song et al in which the SWV measured at 2D SWE/GE was higher than that measured at TE [24]. It is unclear why our results differ from previous research; however, we believe that our results may be more reliable as our study included more types of phantoms than previous studies.

In a previous study regarding diagnostic performance of 2D SWE/GE in hepatic fibrosis staging, the distance between transducer and Glisson capsule was one of the factors affecting its applicability, but it was not a factor affecting the liver stiffness value [36]. It was in accordance with our results. In our study using 4 different SWE platforms, applicability rate and repeatability tends to decrease in phantoms with a thick abdominal wall, but there was no difference in stiffness value according to the abdominal wall thickness. However, our study demonstrating the effects of abdominal wall thickness on applicability and repeatability were different depending on the SWE platforms.

Our study has several limitations. First, our study was an in vitro study using phantoms. Therefore, although we meticulously created porcine abdominal walls in two different thicknesses, they may not reflect the actual diversity of abdominal wall thicknesses in humans. In addition, it can be different from the actual clinical setting, as we could not simulate other factors affecting the measurement of stiffness values such as ascites and respiration. On the other hand, since all other factors were controlled, the influence of abdominal wall thickness may be better demonstrated. Second, we used five non-standardized phantoms of which absolute stiffness values were not provided. If we had used standardized phantoms, we might have determined which elastography method may have been more exact. Third, the mean values of the five phantoms used in our study were $3.6 \mathrm{kPa}, 14.5 \mathrm{kPa}, 16.1$ $\mathrm{kPa}, 16.7 \mathrm{kPa}$ and $18.4 \mathrm{kPa}$, which were measured using TE, the most validated elastography tool, and we could not include a phantom with stiffness values for interme- 
diate fibrosis, according to a meta-analysis of TE for the detection of hepatic fibrosis [37]. Lastly, we only used convex probes for the three SWE platforms and an M probe for TE. If we had measured stiffness values using a linear probe or an XL probe, our results may have been different.

In conclusion, despite the excellent reproducibility of each of the four SWE platforms, we found significantly different applicability, repeatability and stiffness measurements among the platforms. Furthermore, applicability and repeatability of some of the SWE platforms would be more affected by abdominal wall thickness than other SWE platforms. Although 2D SWE/SSI had the highest repeatability, other SWE platforms would be recommended in patients with a thick abdominal wall because the applicability of 2D SWE/SSI was more affected by abdominal wall thickness. Thus, when evaluating liver fibrosis, the appropriate SWE platform could be selected taking into consideration the abdominal thickness of the patients.

\section{Conflict of interest: none}

Acknowledgments: We thank Ms. Yoon Hee Lee and Ms. Mi Ran Lee from Seoul National University Hospital for measuring stiffness values of transient elastography. We also thank Chris Woo for his editorial assistance in preparing this manuscript.

\section{Reference}

1. Yano M, Kumada H, Kage M, et al. The long-term pathological evolution of chronic hepatitis C. Hepatology 1996;23:1334-1340.

2. European Association for Study of Liver. EASL Recommendations on Treatment of Hepatitis C 2015. J Hepatol 2015;63:199-236.

3. Sarin SK, Kumar M, Lau GK, et al. Asian-Pacific clinical practice guidelines on the management of hepatitis B: a 2015 update. Hepatol Int 2016;10:1-98.

4. Calvaruso V, Craxi A. Why do I treat my patients with mild hepatitis C? Liver Int 2016;36 Suppl 1:7-12.

5. Hezode C. Why I do not treat patients for mild disease. Liver Int 2016;36 Suppl 1:13-20.

6. 6.Conti F, Serra C, Vukotic R, et al. Accuracy of elastography point quantification and steatosis influence on assessing liver fibrosis in patients with chronic hepatitis C. Liver Int 2017;37:187-195.

7. Bedossa P, Dargere D, Paradis V. Sampling variability of liver fibrosis in chronic hepatitis C. Hepatology 2003;38:1449-1457.

8. Maharaj B, Maharaj RJ, Leary WP, et al. Sampling variability and its influence on the diagnostic yield of percutaneous needle biopsy of the liver. Lancet 1986;1:523-525.
9. Piscaglia F, Salvatore V, Mulazzani L, Cantisani V, Schiavone C. Ultrasound Shear Wave Elastography for Liver Disease. A Critical Appraisal of the Many Actors on the Stage. Ultraschall Med 2016;37:1-5.

10. Friedrich-Rust M, Poynard T, Castera L. Critical comparison of elastography methods to assess chronic liver disease. Nat Rev Gastroenterol Hepatol 2016;13:402-411.

11. You MW, Kim KW, Pyo J, et al. A Meta-analysis for the Diagnostic Performance of Transient Elastography for Clinically Significant Portal Hypertension. Ultrasound Med Biol 2017;43:59-68.

12. Friedrich-Rust M, Ong MF, Martens S, et al. Performance of transient elastography for the staging of liver fibrosis: a meta-analysis. Gastroenterology 2008;134:960-974.

13. Chang PE, Lui HF, Chau YP, et al. Prospective evaluation of transient elastography for the diagnosis of hepatic fibrosis in Asians: comparison with liver biopsy and aspartate transaminase platelet ratio index. Aliment Pharmacol Ther 2008;28:51-61.

14. European Association for Study of Liver, Asociacion Latinoamericana para el Estudio del Higado. EASL-ALEH Clinical Practice Guidelines: Non-invasive tests for evaluation of liver disease severity and prognosis. J Hepatol 2015;63:237-264.

15. Berzigotti A, Castera L. Update on ultrasound imaging of liver fibrosis. J Hepatol 2013;59:180-182.

16. Shiina T, Nightingale KR, Palmeri ML, et al. WFUMB guidelines and recommendations for clinical use of ultrasound elastography: Part 1: basic principles and terminology. Ultrasound Med Biol 2015;41:1126-1147.

17. Barr RG, Ferraioli G, Palmeri ML, et al. Elastography Assessment of Liver Fibrosis: Society of Radiologists in Ultrasound Consensus Conference Statement. Radiology 2015;276:845-861.

18. Nightingale K, Soo MS, Nightingale R, Trahey G. Acoustic radiation force impulse imaging: in vivo demonstration of clinical feasibility. Ultrasound Med Biol 2002;28:227-235.

19. Cassinotto C, Lapuyade B, Mouries A, et al. Non-invasive assessment of liver fibrosis with impulse elastography: comparison of Supersonic Shear Imaging with ARFI and FibroScan(R). J Hepatol 2014;61:550-557.

20. Yamanaka N, Kaminuma C, Taketomi-Takahashi A, Tsushima Y. Reliable measurement by virtual touch tissue quantification with acoustic radiation force impulse imaging: phantom study. J Ultrasound Med 2012;31:1239-1244.

21. Shin HJ, Kim MJ, Kim HY, Roh YH, Lee MJ. Comparison of shear wave velocities on ultrasound elastography between different machines, transducers, and acquisition depths: a phantom study. Eur Radiol 2016;26:3361-3367.

22. Kohlhaas A, Durango E, Millonig G, et al. Transient elastography with the XL probe rapidly identifies patients with nonhepatic ascites. Hepat Med 2012;4:11-18.

23. Bercoff J, Tanter M, Fink M. Supersonic shear imaging: a new technique for soft tissue elasticity mapping. IEEE Trans Ultrason Ferroelectr Freq Control 2004;51:396-409.

24. Song P, Zhao H, Manduca A, Urban MW, Greenleaf JF, Chen S. Comb-push ultrasound shear elastography (CUSE): 
a novel method for two-dimensional shear elasticity imaging of soft tissues. IEEE Trans Med Imaging 2012;31:18211832.

25. Song P, Urban MW, Manduca A, Zhao H, Greenleaf JF, Chen S. Comb-push ultrasound shear elastography (CUSE) with various ultrasound push beams. IEEE Trans Med Imaging 2013;32:1435-1447.

26. Song P, Macdonald M, Behler R, et al. Two-dimensional shear-wave elastography on conventional ultrasound scanners with time-aligned sequential tracking (TAST) and comb-push ultrasound shear elastography (CUSE). IEEE Trans Ultrason Ferroelectr Freq Control 2015;62:290-302.

27. Yoon JH, Lee JM, Woo HS, et al. Staging of hepatic fibrosis: comparison of magnetic resonance elastography and shear wave elastography in the same individuals. Korean J Radiol. 2013;14:202-212.

28. Castera L, Foucher J, Bernard PH, et al. Pitfalls of liver stiffness measurement: a 5-year prospective study of 13,369 examinations. Hepatology 2010;51:828-835.

29. Poynard T, Munteanu M, Luckina E, et al. Liver fibrosis evaluation using real-time shear wave elastography: applicability and diagnostic performance using methods without a gold standard. J Hepatol 2013;58:928-935.

30. Yoo H, Lee JM, Yoon JH, Lee DH, Chang W, Han JK. Prospective Comparison of Liver Stiffness Measurements between Two Point Shear Wave Elastography Methods: Virtual Touch Quantification and Elastography Point Quantification. Korean J Radiol 2016;17:750-757.
31. Sporea I, Bota S, Gradinaru-Tascau O, Sirli R, Popescu A. Comparative study between two point Shear Wave Elastographic techniques: Acoustic Radiation Force Impulse (ARFI) elastography and ElastPQ. Med Ultrason 2014;16:309-314.

32. Swan KZ, Nielsen VE, Bibby BM, Bonnema SJ. Is the reproducibility of shear wave elastography of thyroid nodules high enough for clinical use? A methodological study. Clin Endocrinol (Oxf) 2017;86:606-613.

33. Mulabecirovic A, Vesterhus M, Gilja OH, Havre RF. In Vitro Comparison of Five Different Elastography Systems for Clinical Applications, Using Strain and Shear Wave Technology. Ultrasound Med Biol 2016;42:2572-2588.

34. Wang CZ, Zheng J, Huang ZP, et al. Influence of measurement depth on the stiffness assessment of healthy liver with real-time shear wave elastography. Ultrasound Med Biol 2014;40:461-469.

35. Potthoff A, Attia D, Pischke S, et al. Influence of different frequencies and insertion depths on the diagnostic accuracy of liver elastography by acoustic radiation force impulse imaging (ARFI). Eur J Radiol 2013;82:1207-1212.

36. Lee SM, Lee JM, Kang HJ, et al. Liver fibrosis staging with a new 2D-shear wave elastography using comb-push technique: Applicability, reproducibility, and diagnostic performance. PLoS One. 2017; 12:e0177264.

37. Stebbing J, Farouk L, Panos G, et al. A meta-analysis of transient elastography for the detection of hepatic fibrosis. J Clin Gastroenterol 2010;44:214-219. 